\title{
The Impact of Interference on the Performance of a Multi-path Metropolitan Wireless Mesh Network
}

\author{
Elias Z. Tragos, Alexandros Fragkiadakis, Ioannis Askoxylakis and Vasilios. A. Siris \\ Institute of Computer Science \\ Foundation for Research and Technology Hellas \\ Heraklion, Greece \\ Email: (etragos)(alfrag)(asko)(vsiris)@ics.forth.gr
}

\begin{abstract}
Wireless mesh networks (WMNs) have attracted much attention lately for providing efficiently wireless services with high quality of service (QoS). Metropolitan WMNs are a low-cost solution for providing broadband wireless internet access in large areas. One of the fundamental issues of wireless communications is interference. In WMNs interference can be caused by simultaneous transmissions at links internal to the mesh network or by external sources. In this work we perform extensive measurements in a multi-radio metropolitan WMN deployed in the city of Heraklion, Greece. The basic goal is to investigate the impact of interference on the performance of the multi-path WMN. Towards that goal, we perform measurements with FTP, video streaming and raw data traffic for two scenarios, one with an efficient channel assignment (CA) that accounts for interference and another with a random $\mathrm{CA}$ that results in high internal and external interference in the network. The results prove that interference creates severe performance degradation, with regards to high delay, high packet losses, low throughput and low signal-to-interference and noise ratio (SINR). As a result, the metropolitan WMN becomes unable to support multi-path flows and demanding applications with an acceptable QoS.
\end{abstract}

\section{INTRODUCTION}

W IRELESS networks have gained increased attention and development during the last decade. The transition from the wired to the wireless world has attracted much research and many solutions have been developed for the efficient operation of the wireless networks, targeting to satisfy the various user needs. Traditional cellular networks like GSM and UMTS are infrastructure networks deployed by operators, which have full control and management of the services offered to the users. In contrast to that, Mobile Ad-hoc Networks are standalone networks, self-organized and built on-the-fly by the users themselves. Wireless Mesh Networks (WMNs), deployed in a joint ad-hoc and infrastructure mode, are an emerging paradigm of networking in an attempt to provide better wireless services to the users [1].

Large networks consisting of many nodes are always difficult to be managed and configured manually. Thus, wireless mesh networks, that normally consist of many nodes, are mainly dynamically self-managed and self-configured, in order

This work was supported in part by the European Commission in the 7th Framework Programme through project EU-MESH (Enhanced, Ubiquitous, and Dependable Broadband Access using MESH Networks), ICT-215320, http://www.eu-mesh.eu

Corresponding author: E. Z. Tragos, tel.: +30 2810 391566, email: etragos@ics.forth.gr to enable the nodes to establish connectivity with the other network nodes, configure themselves automatically and react accordingly to any network problems, by re-configuring their connectivity. The lack of a backbone network infrastructure, automatic configuration, easy management, robustness of the mesh deployment and the low cost of the devices have emerged the WMNs as a reliable and easily-deployed networking solution for providing broadband wireless services with efficient Quality of Service (QoS) to the users. There is a large number of deployment scenarios for WMNs, including broadband home networking, metropolitan and community networking (especially for developing countries, where there is lack of wired infrastructure), business or educational networking (eliminating the need to bury cables in old buildings and across campuses), networking in temporary venues, etc [2], [3].

Normally a WMN consists of several mesh gateways (MGs) for connecting the network with the global Internet and a large group of mesh routers (MRs) and mesh clients (MCs). Since the 802.11 PHY permits simultaneous operation of multiple non-overlapping channels, the mesh routers can be equipped with more than one radio interfaces and communicate with each other with minimum interference, increasing the capacity of the network [4]. The mesh routers act like Access Points providing the routing functions to support the mesh networking and they are usually equipped with multiple wireless interfaces of the same or different access technologies, enabling the integration of the mesh network with other wireless networks, such as wireless sensor networks, WiFi, WiMax, etc. The mesh clients are connected wirelessly to the mesh routers and communicate with other mesh clients in an ad-hoc manner or with the global Internet [1], [2], [3].

Wireless multi-radio multi-channel mesh networks have the potential to provide ubiquitous and high-speed broadband access in urban and rural areas, to both fixed and mobile users, with low operation and management costs. Metropolitan mesh networks have been deployed in several areas, since they are a low-cost solution for providing wireless broadband internet access to large areas. Metropolitan long-distance networks include WiLDNet [5], Roofnet [6], a metropolitan WMN by Rice University [7], etc. Many mesh networks have been deployed in the developing countries, where there is lack of wired infrastructure, i.e. Peebles in South Africa http://www.fmfi.org.za/wiki/images/c/c7/FMFI_Peebles_Valley_Mesh_Dissemination.pdf. 
To investigate issues related to the management and performance of a multi-radio mesh network in an actual metropolitan environment, we have deployed an experimental multi-radio mesh network that covers an area of approximately $60 \mathrm{Km}^{2}$ in the city of Heraklion, Crete, Greece. This paper describes the investigation of the impact of interference on the performance of the multi-path multi-radio metropolitan wireless mesh network. We are taking into account both the external interference from other sources and the internal interference from the links of the WMN. The scope is to prove experimentally that deploying a wireless mesh network in a city without taking into account the interference will result to a poor network performance. Therefore, an efficient channel assignment (CA) algorithm is necessary to minimize interference at the links and its effects on the performance of the mesh network. The rest of the paper is structured as follows: in section 2, we analyze the details of the effect of the interference on wireless links and the channel assignment that we used in our experiments, in section 3 we present the topology of our metropolitan WMN and a description of the experiments, in section 4 we present our experimental results and section 5 concludes the paper.

\section{INTERFERENCE IN WIRELESS MESH NETWORKS}

\section{A. Overview of Interference in wireless networks}

Due to the nature of the wireless medium, interference is a fundamental issue of wireless networks, since the transmissions of one client are interfering with the transmissions or the receptions of the other clients. Controlling interference is essential to achieve maximum performance, since it affects directly the reception capabilities of the clients [8]. Interference is a key factor that can lead to reduced capacity and performance, since it can reduce the achievable transmission rate of wireless interfaces, increase the frame loss ratio and reduce the utilization of the wireless resources, due to contention in distributed channel access protocols, such as IEEE 802.11. Indeed, interference can be between links belonging to the same network or can originate from external sources [9]. Many mechanisms should take into account interference for their efficient functionality, including channel assignment, power control, routing, and network management.

The capacity of WMNs is mainly constrained by the interference as analyzed in [10]. In the absence of interference, a link should provide its maximum capacity, which is determined by the transmission rate and the delivery ratio of the link. Interference is affecting both the sender and the receiver of a link. The sender is transmitting with less than its maximum transmission rate and the receiver has higher probability to unsuccessful packet reception. As described in [10] an analytical model should consider interference in both sides, namely the "sender side", since it causes the sender to defer transmission and the "receiver side" creating collisions when the node receives packets from two simultaneous transmissions. In multi-hop mesh networks the performance degrades as the number of hops increases, since interference is caused in each hop and, in addition to that, in single-radio networks, only one of 2 adjacent hops can be active at any given time. [11]. Multi-radio WMNs have been deployed for increasing the capacity of the network. Every mesh router is equipped with more than one radio interfaces, but still, efficient CA should be performed in order to avoid interference between the interfaces of each node.

There is a variety of factors that are causing interference in mesh networks. In [12] they are split into three categories. The "Intentional Interferers" are transmitting in the same band, in the same area and the signal has the same characteristics with the desired signal. This creates contention for accessing the wireless link, since 802.11 protocol does not allow nodes to transmit simultaneously. This results in nodes waiting some time in order to transmit and thus throughput is reduced. The "Non-intentional Interferers" are transmitting in the same band and area, but their signal is different than the desired and could be from bluetooth, cordless phones, etc. These transmissions are affecting the mesh transmissions by either damaging the packets or not allowing nodes to transmit. The "Multi-path fading" is observed when the received signal comes from two or more different paths, due to reflection, diffraction and scattering. These result in receiving multiple copies of the signal with a delay and with less signal strength, degrading the SINR at the receiver.

In addition to the above, interference is also caused by wireless transmissions not only at the same band, but also at adjacent bands, affecting severely the performance of a wireless multi-radio mesh network. As described in [13], neighboring channels in 802.11a have such power overlap that produces significant interference, decreasing the network throughput. It is essential, though, that in a multi-radio WMN, the radio interfaces of each mesh router are assigned channels that are not interfering with each other. The use of directional antennas can reduce interference between links of a wireless mesh network, since the antennas can beamform towards the intended receivers, which will receive only the desired transmission. The use of directional antennas has many advantages: a) each node can selectively receive signals from a desired direction and avoid interference from other directions, b) increased simultaneous communication, since more users could utilize the network simultaneously, as they will not interfere with each other or content for accessing the wireless link, c) by exploiting the gain of directional antennas, multihop transmissions can be reduced to a minimum number of hops or even to a single-hop transmission [14]. In a multiradio $\mathrm{WMN}$ the use of directional antennas can still create interference between the radio interfaces in each node, since the antennas are quite close to each other and their beams may overlap and thus efficient CA should be performed.

\section{B. Channel Assignment for Minimizing Interference}

Extrapolating from the above, interference is a major factor that reduces the performance of a wireless mesh network. Channel assignment is one of the basic mechanisms than minimize interference in a network. CA in WMNs influences the contention among wireless links and the network topology or connectivity between mesh nodes. Indeed, there is a trade 
off between minimizing the level of contention and maximizing connectivity. Moreover, CA determines the interference between adjacent channels and such interference exists not only for $802.11 \mathrm{~b} / \mathrm{g}$, but also for $802.11 \mathrm{a}$, when the distance of antennas is small, which is the case, when they are located in the same mesh node. Finally, channel assignment influences the connectivity of mesh nodes with wired network gateways, which is a key application of WMNs [15].

In the literature, many channel assignment algorithms for WMNs have been proposed, each of them having specific objectives. For extracting the results in this paper and investigating the impact of interference in a multi-path metropolitan wireless mesh network, we use the algorithm presented in [9], which was built for and tested in the metropolitan mesh network that we use in our experiments. This algorithm introduces the interference-aware concept in the network deployment. It uses a multi-point conflict graph (MPLCG) that is appropriate for network with an a-priori known communication graph (such as our mesh test-bed) and it models effectively the interference between the links of the mesh network. This CA algorithm accounts for both internal and external interference and assigns channels to the links in order to maximize network throughput or minimize the packet delay at the links. The order in which the links are assigned the channels is important and we consider the distance of the links from the gateways, since we assume that these links are more important, as they concentrate the traffic to and from the wired network. Moreover, since MPLCG only models the interference between the links that belong to the mesh network, in order to take into account also the external interference, it is important to use a metric that can capture all types of interference. The SINR as a metric can capture the interference due to external sources and adjacent channel transmissions, but it cannot capture the MAC-layer contention between links that are using the same channel. The round-trip delay as a metric can capture the external interference, the interference due to adjacent channel transmissions and the co-channel interference. It is essential to take into account all these types of interference for the efficient design of the mesh network and for achieving maximum performance.

\section{TeSt-Bed Deployment And ExPeriments Setup}

For experimenting with the performance of a multi-path multi-radio WMN in an actual metropolitan environment, we use an experimental multi-radio mesh network deployed in the city of Heraklion, Crete, Greece. The mesh network is used as a metropolitan scale test-bed built from commodity components with 1 to $5 \mathrm{Km}$ links. The use of commodity IEEE 802.11 technology can lead to significantly lower costs compared to other technologies, such as IEEE 802.16. The metropolitan WMN covers an area of approximately $60 \mathrm{Km}^{2}$ and currently contains 16 nodes (Figure 1) among which six are core mesh nodes and the rest are used for management and monitoring of the network (not displayed for the simplicity of the figure). Each wireless interface is assigned a static IP address, and the Optimized Link State Routing (OLSR)

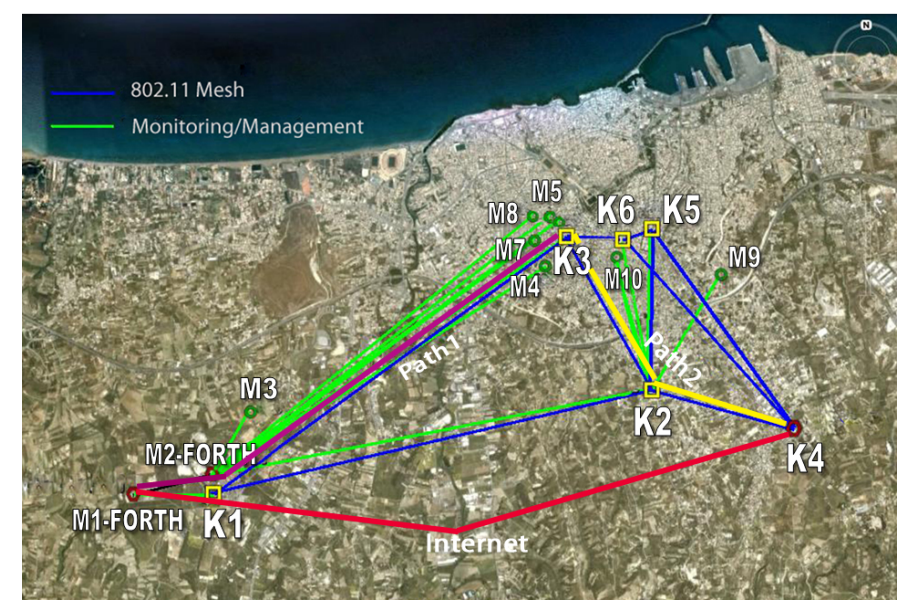

Fig. 1. Topology of the metropolitan mesh network.

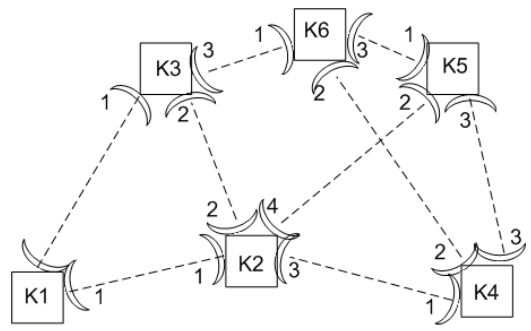

(a) Communication graph

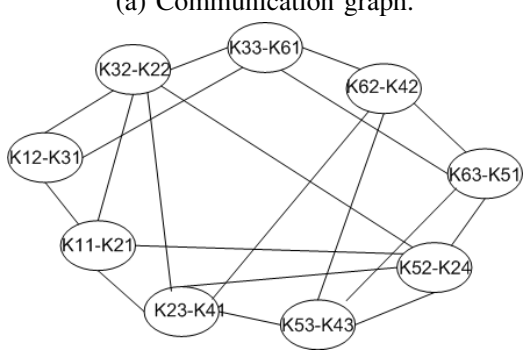

(b) MPLCG.

Fig. 2. Metropolitan WMN's communication graph and MPLCG.

protocol is used for routing traffic. The mesh network is connected to a fixed network via two gateways (nodes $\mathrm{K} 1$ and $\mathrm{K} 4)$. $\mathrm{K} 1$ is connected to the global Internet via M1FORTH and K4 via the University of Crete. In figure 2 the communication graph and the MPLCG of our network is depicted. MPLCG shows the links that are interfering with each other, which corresponds to the lines that are connecting the respective vertices.

The basic goal of the experiments is to test the performance of our network and to show the impact of interference on that performance. Towards this goal, we perform several experiments, which can be split into two basic sets. One set of measurements is taken when the interfaces of the links between the mesh nodes have been assigned channels using the channel assignment algorithm that was presented in the previous section and another set is taken using a random distribution of channels assigned to the interfaces of the mesh 
nodes. Using the channels derived from the CA algorithm, we ensure that the links will not suffer from interference (internal or external), though using the random assignment, we do not avoid interference. We have selected the random assignment and not a fixed assignment with i.e. neighbour links having neighbour channels (which would be almost the worst case scenario) in order, on the one hand to capture random external interference (we cannot know a-priori the external interference) and on the other hand to show that even without the maximum adjacent channel interference, the performance of the network drops significantly.

We perform extensive measurements, but due to space limitations, we present measurements for SINR at node K2 and for end-to-end (e2e) packet delay, e2e throughput and e2e packet loss. These are measured for FTP traffic, raw data traffic (generated using the iperf ${ }^{1}$ tool) and video streaming at node K3. The servers for FTP, video streaming and iperf were located in a PC outside the mesh network. For the synchronization of the clocks of the nodes, which is necessary to get accurate measurements, we have used the "IEEE 1588-2002 Standard for A Precision Clock Synchronization Protocol for Networked Measurement and Control Systems", also known as Precision Time Protocol (PTP) [16], which can achieve a synchronization within 10 microseconds (for regular computers across the internet) or (when the PTP protocol is hardware implemented) down to 10 nanoseconds.

For each test (with minimum or random interference) we perform 3 sets of measurements, 2 for single-path flows and one for a multi-path flow. For the "single-path measurements", the client (node K3) is receiving traffic from the server by a pre-determined single-path route (from either gateway K1 or gateway $\mathrm{K} 4$ for each one of the two single-path scenarios). For the "multi-path measurements", the client K3 is receiving data traffic from the server simultaneously from the 2 different single-paths. By taking single-path and multi-path measurements, we show that interference can affect dramatically the performance of not only a single-path WMN, but also of a multi-path WMN, though on the contrary, efficient channel assignment can also benefit from the advantages of the use of a multi-path for increasing the total throughput and the overall performance of the WMN.

\section{Performance Results}

In this section, the results of the experiments are presented. It must be stated here that in order to perform realistic measurements, we added background traffic to all links of the mesh network, in order to create intra-network interference. We use medium background traffic of around 200kbps, with the command line tool "ping" in each node, sending packets of size 1400bytes every $100 \mathrm{~ms}$ to each neighbor node. As mentioned previously, the experiments include scenarios for FTP traffic, video streaming and iperf traffic. For FTP, the transferred file has a size of around $45 \mathrm{MB}$, for video streaming, we stream for 75 seconds a video with a $2 \mathrm{Mbps}$

\footnotetext{
${ }^{1}$ http://iperf.sourceforge.net/
}

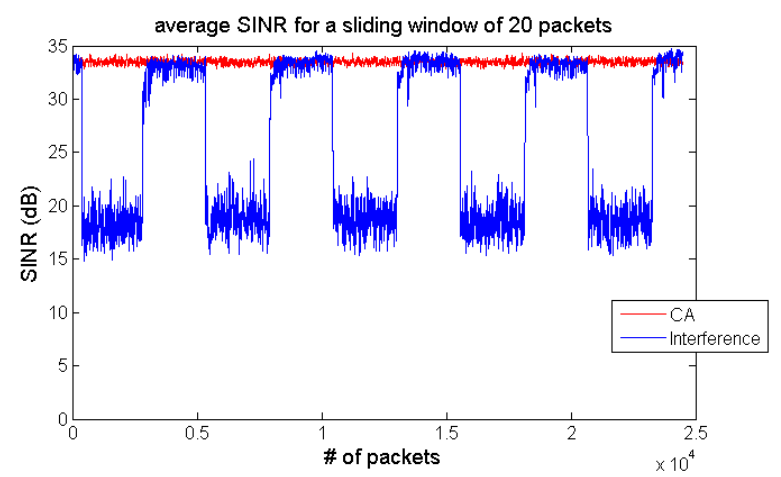

Fig. 3. SINR measurements at node K2.

datarate demand and for iperf traffic, we send 14Mbps UDP traffic for 60 seconds. It should be noted here that, during our experiments, the OLSR protocol was disabled and we used static routing at the mesh nodes.

Figure 3 depicts the measured signal to interference plus noise ratio (SINR) at the interface of node $\mathrm{K} 2$ that is connected to node K3. For this experiment we create constant traffic in the K2-K3 link and periodic traffic in all the other links. In the case of the channel assignment algorithm, the SINR is not affected by the periodic traffic, which means that, despite the traffic, no interference is created between the links of the network. In the case of the random assignment, the SINR drops significantly when there is traffic in the other links, which means that the other links are interfering with the observed link and this interference is causing a huge drop of the SINR to almost the half of the value that is measured, when there is no traffic in the other links.

In the following tables, the rows labelled "Channel Assignment" include the measurements at the case when the interfaces of the mesh nodes have been assigned channels by the channel assignment algorithm that accounts for both internal and external interference and the rows labelled "Interference" present the results with the random assignment that does not take into account the interference for assigning channels to the interfaces, which results in higher interference either between the links or external from other sources.

Table I shows the mean e2e delay values for 5 different measurements for FTP and video streaming traffic for the two cases (with minimum or higher interference) and for the three different scenarios, namely the two single-path scenarios and the multi-path scenario that sends traffic to node K3 from both single-path routes. As it is obvious, interference results in much higher mean e2e packet delay for both FTP and video streaming traffic. As we can see in the "Delay Increase" rows (where the percentage of the increase of the e2e delay when high interference exist is displayed), interference causes a significant increase in the e2e packet delay that varies from 5\% (for FTP single-path through K1) to over $73 \%$ (for FTP single-path through K4 and K2). We should note here the huge difference in the single-path K4-K2-K3, where the mean packet delay is more than double (in the 
TABLE I

MEAN DELAY

\begin{tabular}{|l|c|c|c|}
\hline & \multicolumn{3}{|c|}{ packet Delay for FTP traffic(ms) } \\
\hline Scenario & path K1-K3 & path K4-K2-K3 & Multi-path \\
\hline Channel Assignment & 12.437 & 19.215 & 13.587 \\
\hline Interference & 13.061 & 33.273 & 15.983 \\
\hline Delay Increase & $\mathbf{5 . 0 1 \%}$ & $\mathbf{7 3 . 1 9 \%}$ & $\mathbf{1 7 . 6 3 \%}$ \\
\hline & \multicolumn{2}{|c|}{ packet Delay for Video traffic (ms) } \\
\hline Scenario & path K1-K3 & path K4-K2-K3 & Multi-path \\
\hline Channel Assignment & 6.137 & 11.937 & 8.766 \\
\hline Interference & 8.652 & 13.577 & 10.574 \\
\hline Delay Increase & $\mathbf{4 0 . 9 8 \%}$ & $\mathbf{1 3 . 7 4 \%}$ & $\mathbf{2 0 . 6 3 \%}$ \\
\hline
\end{tabular}

case of FTP) when there is high interference in the links. This shows exactly the huge impact that interference has on a multi-hop link in a mesh network and can be explained in two ways. First, the multi-hop link is composed of the two links $\mathrm{K} 3-\mathrm{K} 2$ and $\mathrm{K} 2-\mathrm{K} 4$ and the external interference at each link is being summed up and impacts the total performance of the multi-path link. Moreover, as seen in Figure 2b, links "K32-K22" and "K23-K41" that consist the multi-hop link, are interfering with four and five other links of the mesh network respectively, creating much internal interference to the multi-hop link, resulting to much higher e2e delay. This happens because interference causes either longer waiting time for transmissions (the transmitters see the band occupied for longer time) or packet retransmissions due to low received SINR. In the multi-path case, it is obvious that when there is high interference in both paths, the delay is much higher and it reaches $20 \%$ in the case of video streaming traffic. Comparing the single-path and the multi-path scenarios, we can see that in the latter case, delay is higher than in the path through K1 and less than in the path through K4-K2. This means that if we are interested only in keeping the e2e delay very low, the multi-path scenario is not profitable.

Table II shows the mean throughput values for 5 different measurements for FTP and iperf traffic for the two cases (with minimum and high interference), for the two singlepaths and the multi-path scenario. The "Degradation" rows show the percentage of the throughput decrease, due to the existence of high interference in the links with the use of the random channel assignment. The mean throughput is quite lower when high interference exists and the percentage of the decrease varies from $14 \%$ (iperf traffic and singlepath through K4) down to 53\% (FTP traffic and multi-path). Regarding the single-path scenarios, the loss of throughput due to high interference is quite significant in the case of FTP traffic through the multi-hop path, where the throughput drops almost to half of that achieved using the channel assignment algorithm. An important observation is the significant impact of interference in the multi-path scenario. One would assume that using two paths for receiving traffic would benefit a user and the received throughput would be much higher comparing to the one achieved by using one path only. This is not valid when the channels assigned in the wireless links are not derived by an efficient algorithm, which results to high interference in the links. We see from the results that the
TABLE II

MEAN THROUGHPUT

\begin{tabular}{|l|c|c|c|}
\hline & \multicolumn{3}{|c|}{ FTP Throughput (Mbps) } \\
\hline Scenario & path K1-K3 & path K4-K2-K3 & Multi-path \\
\hline Channel Assignment & 7.304 & 9.188 & 14.712 \\
\hline Interference & 6.047 & 5.045 & 6.901 \\
\hline Degradation & $\mathbf{1 7 . 2 1 \%}$ & $\mathbf{4 5 . 0 9 \%}$ & $\mathbf{5 3 . 0 9 \%}$ \\
\hline & \multicolumn{3}{|c|}{ iperf Throughput (Mbps) } \\
\hline Scenario & path K1-K3 & path K4-K2-K3 & Multi-path \\
\hline Channel Assignment & 12.24 & 13.92 & 14.24 \\
\hline Interference & 9.76 & 11.92 & 10.96 \\
\hline Degradation & $\mathbf{2 4 . 3 4 \%}$ & $\mathbf{1 4 . 3 7 \%}$ & $\mathbf{2 3 . 0 3 \%}$ \\
\hline
\end{tabular}

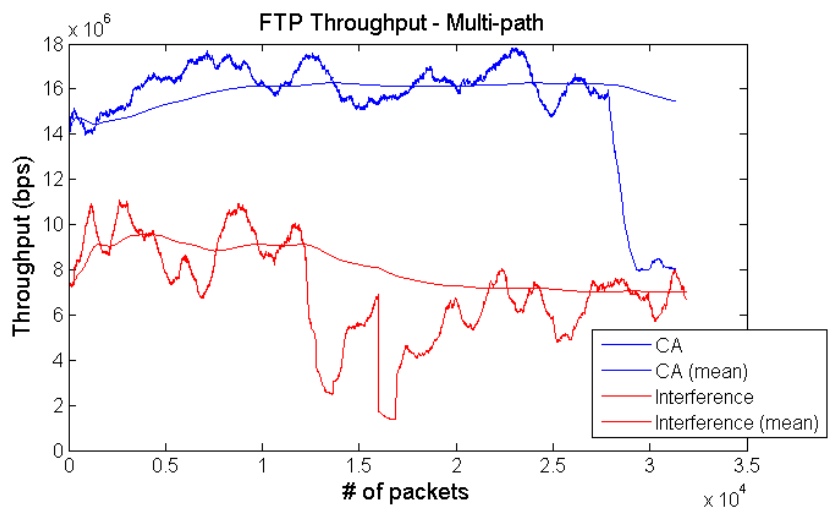

Fig. 4. FTP throughput at node K3 for multi-path.

achieved throughput in the multi-path scenario in both FTP and iperf traffic is quite low. In fact, in the case of iperf traffic, the achieved multi-path throughput is even lower than in the case of the single-path through K4. In the case of FTP traffic, the multi-path throughput is slightly higher than in the singlepath through K1. Contrary to that, when the interference in the links is minimized using the channel assignment algorithm, we can see that in the FTP traffic the multi-path throughput is much higher than in the single-path cases (over $40 \%$ higher) and in the iperf traffic is also quite high. As a result, in a metropolitan WMN, the multi-path can benefit users to achieve higher throughput, only when the links are configured in a way to minimize interference, otherwise the resulting throughput may be even lower than the one achieved via a single-path.

In Figure 4 the FTP throughput of one run of measurements for the multi-path case for both the CA and the random assignment scenario is depicted. It shows the actual throughput per packet and the mean throughput until the time that this packet was received, with a sliding window of $5 \mathrm{sec}$. This figure is another example that proves how much interference impacts the network throughput in the multi-path scenario.

Table III shows the average packet loss measured in the experiments for video streaming and iperf. In the iperf case, interference in the links results in at least the triple percentage of lost packets and in the multi-path case a huge difference was observed. In the case of video traffic our channel assignment algorithm results in a packet loss ratio much lower than $0.01 \%$ in all cases. On the contrary, when there is interference in the links, the packet loss ratio increases dramatically for all 
TABLE III

PACKET LOSS

\begin{tabular}{|l|c|c|c|}
\hline & \multicolumn{4}{|c|}{ Video Streaming - lost packets (percentage-absolute) } \\
\hline Scenario & path K1-K3 & path K4-K2-K3 & Multi-path \\
\hline Ch. As. & $0.0036 \%(0.6)$ & $0.0036 \%(0.6)$ & $0.0073 \%(1.2)$ \\
\hline Interference & $0.885 \%(146.4)$ & $0.053 \%(8.8)$ & $0.878 \%(145.2)$ \\
\hline Increase & $\mathbf{2 4 3 0 0 \%}$ & $\mathbf{1 3 6 6 . 6 6 \%}$ & $\mathbf{1 2 0 0 0 \%}$ \\
\hline & \multicolumn{2}{|c|}{ iperf - lost packets (percentage) } \\
\hline Scenario & path K1-K3 & path K4-K2-K3 & Multi-path \\
\hline Ch. As. & $13.09 \%$ & $0.27 \%$ & $0.16 \%$ \\
\hline Interference & $31.7 \%$ & $15.34 \%$ & $25.29 \%$ \\
\hline Increase & $\mathbf{1 4 2 . 1 7 \%}$ & $\mathbf{5 5 8 1 . 4 8 \%}$ & $\mathbf{1 5 7 0 6 . 2 5 \%}$ \\
\hline
\end{tabular}

scenarios and this results in a packet loss percentage from 0.053 to 0.885 . It should be noted here that, for H.264 video codecs (which was the one that we used for our video), a maximum of $0.1 \%$ packet loss is recommended for video streaming without error concealment and/or correction, as mentioned in [17]. As shown in the table, the mesh network can support the video without any problems only in the case of the channel assignment algorithm. Interference causes a packet loss ratio of around $0.8 \%$ (even in the multi-path case), which is much higher than the acceptable ratio of $0.1 \%$. This results in unacceptable video quality, degrading the quality of user experience (QoE).

\section{CONClusion}

In this paper we discuss the problem of interference in an experimental multi-path metropolitan wireless mesh network. Interference is one of the most important issues in wireless communications, since it causes problems in the transmissions, degrading the quality of the communications. We have tested the performance of the mesh network in two scenarios; one using random assignment of channels to the wireless interfaces of the mesh nodes, which results in high interference in the links and another by using a channel assignment algorithm that accounts for both internal and external interference, which minimizes the interference in the links. The experiments proved that interference can severely degrade the performance of a mesh network, not only for single-path, but also for multi-path flows. The presence of interference was also shown by the SINR measurements, where a significant drop of the received SINR was observed when there was light traffic in the network in the random assignment case. Using efficient channel assignment, interference in the network is minimized and the clients can furthermore benefit from the use of two internet gateways in the multi-path scenario, achieving almost double throughput, in comparison to the one achieved in the presence of high interference between the links, keeping at the same time the overall packet delay very low. A very interesting outcome was that the existence of interference in the mesh network results in a very high percentage of lost packets and makes the mesh network incapable of delivering a video streaming service with the required quality either in the single-path or in the multi-path scenario. The multi-path scenario would be expected to benefit the user to achieve higher performance, in terms of higher throughput and video quality. In contrast to that, our experiments proved that an inefficient channel assignment algorithm can result in such high interference, not only from external sources, but also between the mesh network's links, such that the multi-path flows would achieve very low overall throughput, very high packet losses and very high delay, comparing to the single path flows of the efficient channel assignment. In general, the results proved that a metropolitan mesh network, even with the use of directional antennas, should be very carefully configured with a proper channel assignment algorithm, in order to achieve its maximum performance and exploit the use of multiple gateways and multiple paths for increasing the maximum received throughput of the users.

\section{REFERENCES}

[1] H. Moustafa, U. Javaid, D. E. Meddour and S. M. Senouci, A Panorama on Wireless Mesh Networks: Architectures, Applications and Technical Challenges, Proc. of Wimeshnets, Waterloo, Canada, Aug'06.

[2] I. F. Akyildiz and X. Wang, A Survey on Wireless Mesh Networks, IEEE Communications Magazine, vol. 43, no. 9, s23-s30, Sept. 2005

[3] I. F. Akyildiz, X. Wang, W. Wang, Wireless mesh networks: a survey. Computer Networks and ISDN Systems v.47 n.4. pp. 445487, 15 March 2005.

[4] K. N. Ramachandran, E. M. Belding, K. C. Almeroth, and M. M. Buddhikot, Interference-aware channel assignment in multi-radio wireless mesh networks, Proc. of IEEE INFOCOM, Barcelona, Spain, April 2006.

[5] R. Patra, S. Nedevschi, S. Surama, A. Sheth, L. Subramanian, and E. Brewer, WiLDNet: Design and Implementation of High Performance WiFi Based Long Distance Networks.Proc. of USENIX Symposium on Networked Systems Design and Implementation (NSDI), 2006.

[6] J. Bicket, D. Aguayo, S. Biswas, and R. Morris, Architecture and Evaluation of an Unplanned 802.11b Mesh Network. In Proc. of ACM MOBICOM, 2005

[7] J. Camp, J. Robinson, C. Steger, and E. Knightly, Measurement Driven Deployment of a Two-Tier Urban Mesh Access Network, In Proc. of ACM MobiSys, 2006.

[8] L. Qiu, Y. Zhang, F. Wang, M. K. Han, and R. Mahajan, A General Model of Wireless Interference, Proc. of MobiCom, September 2007

[9] M. Delakis and V. A. Siris, Channel Assignment in a Metropolitan Wireless Multi-Radio Mesh Network, Proc. of Broadnets, London, September 2008.

[10] A. Kashyap, S. Ganguly and S. Das, Characterizing Interference in 802.11-based Wireless Mesh Networks, Technical Report, 2006

[11] K. Jain, J. Padhye, V. N. Padmanabhan, L. Qiu, Impact Of Interference On Multi-hop Wireless Network Performance, Proc. of Mobicom, San Diego, USA, September 2003.

[12] S. Das, D. Koutsonikolas, Y. C. Hu, D. Peroulis, Characterizing MultiWay Interference In Wireless Mesh Networks, Proc. of WINTECH, Los Angeles, USA, 2006.

[13] V. Angelakis, S. Papadakis, N. Kossifidis, V. A. Siris, A. Traganitis, The Effect of Using Directional Antennas on Adjacent Channel Interference in 802.11a: Modeling and Experience With an Outdoors Testbed, Proc. of WiNMee, Berlin, Germany, March 2008.

[14] S. Kandasamy, R. Campos, R. Morla, M. Ricardo. Using Directional Antennas on Stub Wireless Mesh Networks: Impact on Throughput, Delay, and Fairness, Proc. of IEEE WiMAN 2010, Aug 2010.

[15] T. Dionysiou, V. A. Siris, G. Stamatakis, Utility-based Channel Assignment and Topology Control in Wireless Mesh Networks, Proc. of IEEE WoWMoM, Montreal, Canada, June 2010.

[16] IEEE 1588-2002, Standard for A Precision Clock Synchronization Protocol for Networked Measurement and Control Systems, http://ieee1588.nist.gov/

[17] M. H. Pinson, S. Wolf, and R. B. Stafford, Video Performance Requirements for Tactical Video Applications, IEEE conference on Technologies and Homeland Security, May 2007, Boston, USA. 